\title{
Coevolving Dynamics between Epidemic and Information Spreading considering the Dependence between Vigilance and Awareness Prevalence
}

\author{
Yixiao Zhang, ${ }^{1}$ Xing Lu, ${ }^{1}$ Ni Cui $\mathbb{D},{ }^{1}$ Jingtai Tang $\mathbb{D},{ }^{2}$ and Xiyun Zhang $\mathbb{D}^{1}$ \\ ${ }^{1}$ Department of Physics, Jinan University, Guangzhou, Guangdong 510632, China \\ ${ }^{2}$ School of Journalism and Communication, Jinan University, Guangzhou, Guangdong 510632, China \\ Correspondence should be addressed to Ni Cui; cuinicn@outlook.com, Jingtai Tang; tangjingtai@jnu.edu.cn, and Xiyun Zhang; \\ zxy_822@126.com
}

Received 24 February 2021; Accepted 11 May 2021; Published 24 May 2021

Academic Editor: Chenquan Gan

Copyright (C) 2021 Yixiao Zhang et al. This is an open access article distributed under the Creative Commons Attribution License, which permits unrestricted use, distribution, and reproduction in any medium, provided the original work is properly cited.

It has been demonstrated that the propagation of information and awareness regarding a disease can assist in containing the outbreak of epidemics. Previous models for this coevolving usually introduced the dependence between these two processes by setting a lower but time-independent infection rate for individuals with awareness. However, a realistic scenario can be more complicated, as individual vigilance and the adopted protective measures may depend on the extent of the discussion on the disease, whereas individuals may be irrational or lack relevant knowledge, leading to improper measures being taken. These can introduce a time-varying dependence between epidemic dynamics and awareness prevalence and may weaken the effect of spreading awareness in containing a pandemic. To better understand this effect, we introduce a nonlinear dependence of the epidemic infection rate on awareness prevalence, focusing on the effect of different forms of dependence on the coevolving dynamics. We demonstrate that a positive correlation between vigilance and awareness prevalence can enhance the effect of information spreading in suppressing epidemics. However, this enhancement can be weakened if some individuals are irrational. Our results demonstrate the importance of rational behavior in the strategy of containing epidemics by propagation of disease information.

\section{Introduction}

Fighting a pandemic is a major challenge. Therefore, understanding the dynamics of epidemic spreading and determining an efficient strategy to contain a pandemic have attracted considerable attention in complex networks [1]. Various factors and effects have been discussed, from the network topology and competition between viruses to human behavior and robustness of the health system [2-12]. With the rapid development of modern techniques, the pattern of epidemic spreading has also changed. For instance, the development of modern airline transportation facilitates long-distance travel; however, it also promotes the spread of pathogens, such as the epidemic of SARS [13-15] and the novel influenza $\mathrm{A}\left(\mathrm{H}_{1} \mathrm{~N}_{1}\right)$ [16-18]. High-speed trains are the best choice for intermediate-distance travel in several countries; however, the high passenger density on these trains can also accelerate the spread of viruses [19]. Cold chain logistics has become one of the most important techniques to ensure food supply. Recently, during the COVID-19 pandemic, it was found that this food transport technique can also carry pathogens and cause local epidemic events [20]. Therefore, there is an increasing demand for more realistic models to better understand epidemic spreading.

One of the characteristics of modern living is the omnipresence of information. Reading news, browsing webpages, and participating in online social networks have become important parts of our daily lives. Messages received through these activities can influence our decision making, can change our behaviors, and, as a result, may also impact the pattern of epidemic spreading [21-24]. Therefore, 
understanding the effect of information propagation on the spread of a disease has attracted considerable attention. Models have been proposed to investigate the suppressive effect of spreading awareness on epidemic propagation, focusing on different mechanisms, such as nonlinear coupling [22, 23, 25-29] and competition [30-33].

Recent studies on the coevolution dynamics between information and disease spreading usually assume that the response of individuals to messages is independent of time, and the response to information is manifested by a relatively lower infection rate for individuals with awareness. However, a realistic situation can be more complicated: an individual may not immediately take the highest level of protective measures when he/she receives information regarding the epidemic. The level of protective measures is usually upgraded when individuals become more vigilant or frightened. Recently, it has been demonstrated that this vigilance (or fear) is related to the prevalence of public discussion on epidemics [34-36]. Therefore, it is possible that individuals become more vigilant and upgrade their protective measures when they receive more information regarding the pandemic. That is, there could be a positive correlation between the prevalence of the spreading of awareness of a disease and the protective measures taken by individuals. To better illustrate this, we collected data on COVID-19 in China [37] and the corresponding online discussion on Sina Weibo, the largest microblogging system in China. Figure 1 shows a plot of the number of existing cases of COVID-19 (red line), representing the prevalence of the epidemic, and the number of discussions on the epidemic on Sina Weibo (green line). As disinfection is one of the main protective measures taken by individuals to prevent the spread of epidemics, the prevalence of discussions on disinfection can thus reflect the level of vigilance or fear of the pandemic. Thus, we also indicate the prevalence of discussions on disinfection on Sina Weibo (blue line). As shown in Figure 1, the heat of the discussion changes with the prevalence of the epidemic in general; that is, the heat of the discussion increases quickly when the epidemic starts and gradually decreases after the epidemic is contained. However, the short-term fluctuation does not exactly reflect the trend of the epidemic. For instance, there is a peak in the heat of the discussion on Sina Weibo around the beginning of April 2020, when the number of existing infected cases continuously decreased. This peak was due to the Qingming day, the traditional tomb-sweeping day in China. A memorial service was held this day; thus, an increase in the discussion on Sina Weibo appeared. Nevertheless, the discussion on disinfection, which can represent the level of vigilance of individuals, follows the heat of the discussion on the epidemic. This indicates that the vigilance of individuals does not always follow the development of the epidemic but the heat of the discussion. Therefore, the effect of the dependence between individual vigilance and the prevalence of the discussion on the coevolving dynamics of information and epidemic spreading is worth investigating.

Previous studies on the coevolving dynamics of information and epidemic spreading usually assume that individuals are rational and can take proper protective measures.
However, during a pandemic, not all individuals are rational. Irrational behaviors, such as irrational beliefs [38], irrational antimicrobial prescribing [39], and panic buying [40], cannot protect individuals from the epidemic. On the contrary, these irrational behaviors may facilitate the spread of the epidemic by inducing individuals to take improper protective measures. Therefore, the spread of awareness of the disease can suppress the epidemic when individuals can take proper protective measures. However, it may also facilitate the spread of the disease if individuals are irrational and take inadequate measures. This leads to a negative correlation between the efficiency of individual protective measures and the prevalence of the discussion for irrational individuals.

In this study, based on the framework of the coevolving dynamics of information and disease spreading in two-layer networks, we consider the effect of dependence between individual vigilance and the prevalence of the discussion. Using brief analytics and numerical simulations, we demonstrate that a positive dependence can assist in containing the spread of the epidemic. In some cases, strong individual vigilance can suppress the size of the final outbreak even without increasing the awareness of the population. However, irrational individuals can weaken this suppressive effect. In some extreme cases, when most individuals are irrational, the spreading of information can no longer suppress epidemics.

The remainder of this paper is organized as follows. In Section 2, we introduce the dynamic model. In Section 3, we briefly provide a theoretical analysis. The results for various scenarios are presented in Section 4. The conclusions are presented in Section 5.

\section{Model}

The model contains two components, as shown in Figure 2. The unaware-aware-unaware (UAU) dynamics describe information spreading. An individual can become aware when (i) he/she is infected by the disease or (ii) through communication with aware neighbors (with probability $\lambda$ ). At each step, each individual becomes unaware with probability $\delta$. In the contagion network, a susceptible-infected-susceptible (SIS) model is used to describe the spread of the disease. The infection rates are $\beta^{U}$ and $\beta^{A}$ for individuals with and without awareness, respectively. As an individual will take protective measures when he/she becomes aware of the disease, we assume that $\beta^{A}=\gamma \beta^{U}$, where $0 \leq \gamma \leq 1$ is related to individual vigilance. The recovery rate for the SIS model is $\mu$.

To incorporate the dependence between individual vigilance and the prevalence of awareness of the disease, we now investigate two specific forms of dependence, where $\rho_{A}$ is the ratio of the population with awareness:

$$
\begin{aligned}
& \text { Dependence 1: } \gamma=\left(1-\rho_{A}\right)^{x} \text {, with } x>0 \\
& \text { Dependence 2: } \gamma=x^{-\rho_{A}} \text {, with } x>1
\end{aligned}
$$

Notably, the dynamics of the model are similar to those of the model in [30]. The difference is that we introduce a dependence between the protective efficiency of individuals 


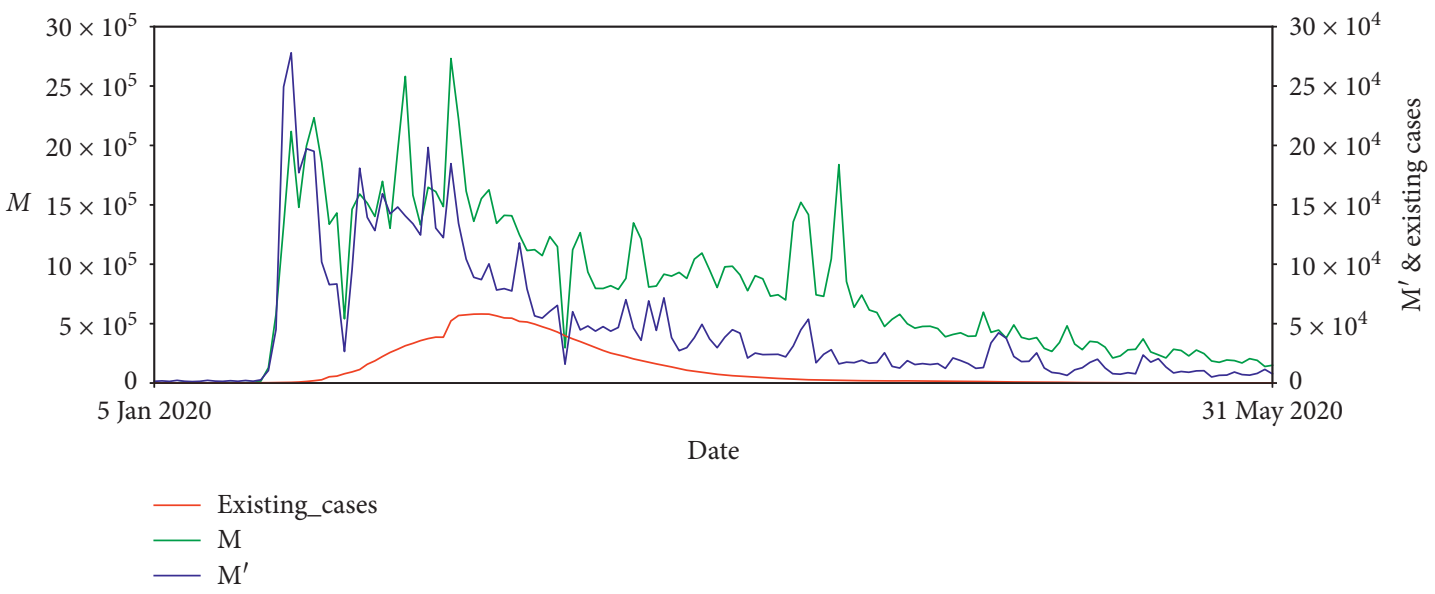

(a)

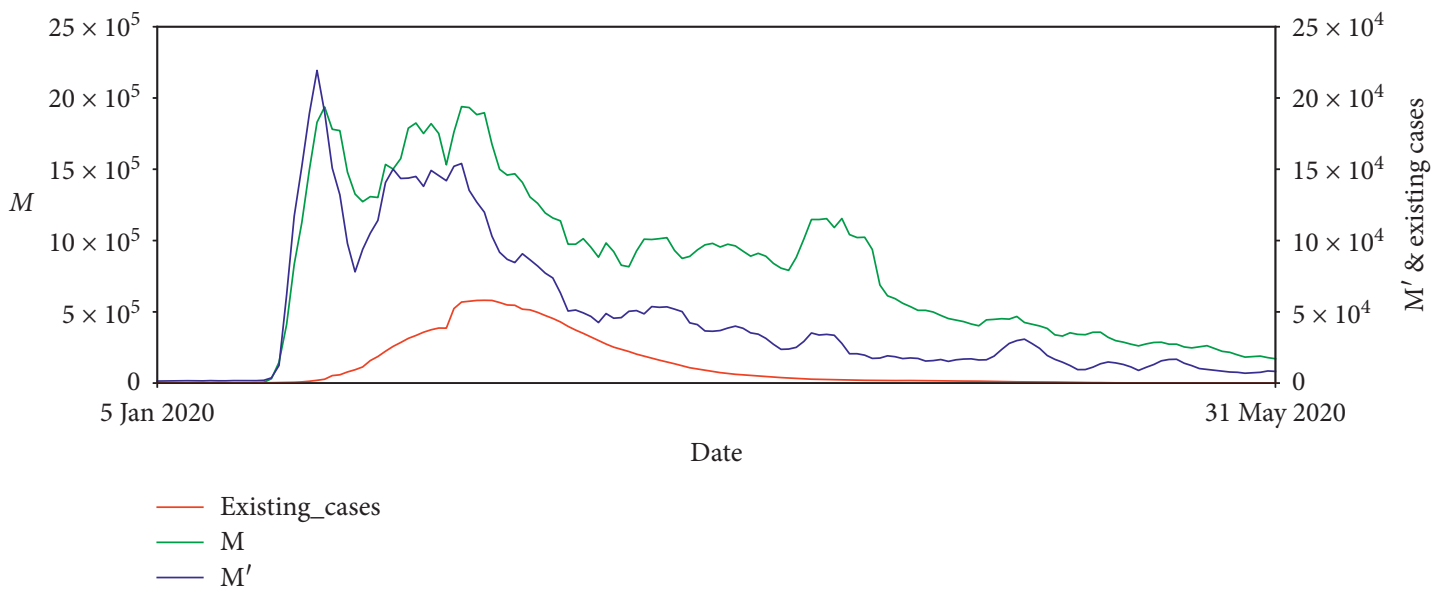

(b)

Figure 1: (a) Number of existing cases of COVID-19 in China (red line), number of discussions about COVID-19 (M) (green line), and disinfection on Sina Weibo (M', blue line). (b) Smoothed curves in (a) using nonoverlapping windows, $w=5$.

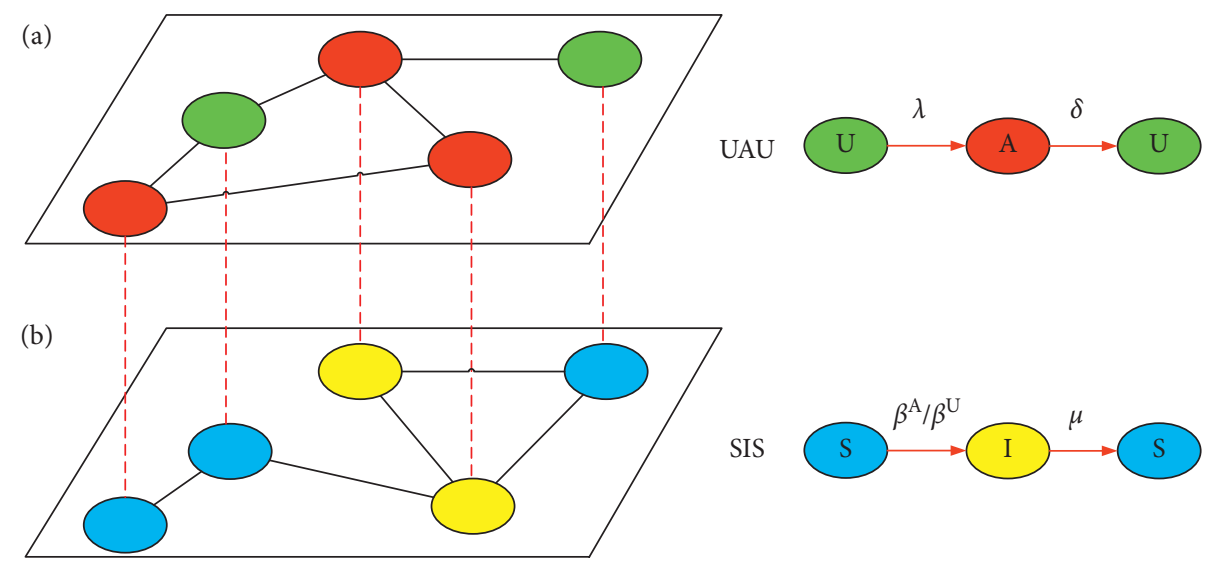

FIGURE 2: Schematic of the model. The dynamics of information spreading are described using the UAU model in the information network. The epidemic spreading process is described by the SIS model in the contagion network. 
and the prevalence of awareness. Therefore, parameter $\gamma$ is not constant, but it changes with the spreading of awareness.

\section{Model Analysis}

In the model, each individual can be unaware $(U)$ or aware $(A)$ and susceptible $(S)$ or infected $(I)$. According to the dynamics, three states are possible in the system: US, AS, and $A I$. We note that the UI state is erroneous because an individual will become aware of the disease immediately when he/she is infected. Here, we only include the correlation between $\rho_{A}$ and $\gamma$; the transition trees for the possible states in our model are the same as those in [30], as shown in Figure 3. Therefore, we can also apply the microscopic Markov chain approach (MMCA) to analyze the coevolution dynamics between information spreading and disease spreading [30, 41-43].

We denote by $a_{i j}$ and $b_{i j}$ the adjacency matrices of the information- and disease-spreading networks, respectively. The probability that node $i$ is in one of the three states at time $t$ is denoted by $p_{i}^{A I}(t), p_{i}^{A S}(t)$, and $p_{i}^{U S}(t)$. The transition probability for node $i$ from unaware to aware is $r_{i}(t)$. The probabilities for the transition from susceptible to infected are $q_{i}^{A}(t)$ and $q_{i}^{U}(t)$ for nodes with and without awareness, respectively. These probabilities can be estimated as

$$
\begin{aligned}
r_{i}(t) & =\prod_{j}\left[1-a_{i j} p_{j}^{A}(t) \lambda\right], \\
q_{i}^{A}(t) & =\prod_{j}\left[1-b_{i j} p_{j}^{A I}(t) \beta^{A}\right], \\
q_{i}^{U}(t) & =\prod_{j}\left[1-b_{i j} p_{j}^{A I}(t) \beta^{U}\right],
\end{aligned}
$$

where $p_{j}^{A}(t)=p_{j}^{A I}(t)+p_{j}^{A S}(t)$. Therefore, according to the transition trees shown in Figure 3 and the transition probabilities calculated using equations (1)-(3), the microscopic Markov chains for the dynamics of each node can be obtained as

$$
\begin{aligned}
p_{i}^{U S}(t+1)= & p_{i}^{A I}(t) \delta \mu+p_{i}^{U S}(t) r_{i}(t) q_{i}^{U}(t)+p_{i}^{A S}(t) \delta q_{i}^{U}(t), \\
p_{i}^{A S}(t+1)= & p_{i}^{A I}(t)(1-\delta) \mu+p_{i}^{U S}(t)\left(1-r_{i}(t)\right) q_{i}^{A}(t)+p_{i}^{A S}(t)(1-\delta) q_{i}^{A}(t), \\
p_{i}^{A I}(t+1)= & p_{i}^{A I}(t)(1-\mu)+p_{i}^{U S}(t)\left\{\left[1-r_{i}(t)\right]\left[1-q_{i}^{A}(t)\right]+r_{i}(t)\left[1-q_{i}^{U}(t)\right]\right\} \\
& +p_{i}^{A S}(t)\left\{\delta\left[1-q_{i}^{U}(t)\right]+(1-\delta)\left[1-q_{i}^{A}(t)\right]\right\} .
\end{aligned}
$$

The stationary state of the system can be estimated by the stationary state in equation (4). The populations of infected nodes and nodes with awareness are defined as $\rho_{I}=(1 / N) \sum_{i} p_{i}^{I}$ and $\rho_{A}=(1 / N) \sum_{i} p_{i}^{A}$, respectively.

\section{Results}

To quantitatively study the effect of different forms of dependence between individual vigilance and the prevalence of the discussion on the spread of the epidemic, we now focus on the two functional forms by comparing the results of a simulation with the theoretical estimation from equation (4). The simulation results were obtained by averaging the results from 30 independent realizations, based on Erdös-Rényi (ER) random networks, with $N=1000$ nodes, and average degree $\langle k\rangle=12$ for the information network and $\langle k\rangle=10$ for the disease network. The recovery rates were set to $\delta=$ 0.6 and $\mu=0.4$. The theoretical estimation was obtained by iterating equation (4) until a stationary state was reached. For simplicity, we define $\beta^{U}=\beta$. The reproduction rate $R_{0}=$ $\left(\beta\left\langle k_{b}\right\rangle / \mu\right)$ is the key factor that determines the spread of the disease. Thus, we focus on the spreading pattern for different $R_{0}$.

As described in the model, individuals with awareness will take protective measures, leading to a reduced infection rate $\beta^{A}=\gamma \beta^{U}$ with $\gamma \leq 1$. Therefore, the reproduction rate $R_{0}$ for individuals with awareness is also reduced. As individual vigilance can change with the prevalence of the discussion about the epidemic, we introduce a function for the dependence between $\gamma$ and $\rho_{A}$. Figure 4(a) shows a plot of the first function $\gamma=\left(1-\rho_{A}\right)^{x}$. As $x$ increases, $\rho_{A}$ decreases faster with an increase in $\rho_{A}$, indicating a faster increase in individual vigilance. As a result, a larger $x$ results in a lower ratio of infected nodes, as well as a lower ratio of nodes with awareness, as shown in Figures 4(b)-4(d). Notably, for large $R_{0}, \rho_{A}$ saturates and does not increase; however, a larger $x$ can suppress the spread of the epidemic owing to the increase in the individual vigilance. Figures 4(e) and 4(f) show the dependence of the critical infection rate $R_{0 c}$ on $x$ and $\lambda$. As in [30], increasing the information propagation rate $\lambda$ holds back the outbreak of the epidemic. In addition, a smaller $x$ can also significantly delay the outbreak of the epidemic, indicating that a positive correlation between individual vigilance and the prevalence of discussion can indeed suppress the outbreak of the epidemic.

Regarding the second form of $\gamma=x^{-\rho_{A}}$, Figure 5(a) shows that the level of vigilance also increases with $x$, as is the case with the first form in Figure 4. Thus, a larger $x$ also leads to a lower ratio of infected population (Figures 5(b)-5(d)) and a larger critical infection rate $R_{0 c}$ (Figures 5(e) and 5(f)). Combining the results of the different forms of dependence between $\gamma$ and $\rho_{A}$ (Figures 4 and 5), we can conclude that a positive correlation between individual vigilance and the prevalence of discussion can significantly assist in containing the epidemic. In addition, when $\rho_{A}$ saturates for large $\beta$, increasing individual vigilance can further suppress the epidemic. 


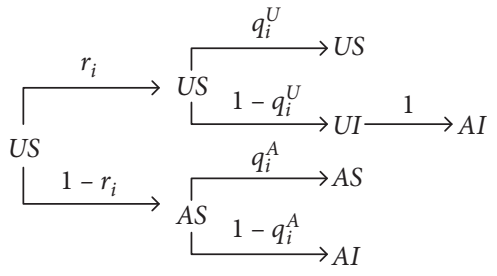

(a)

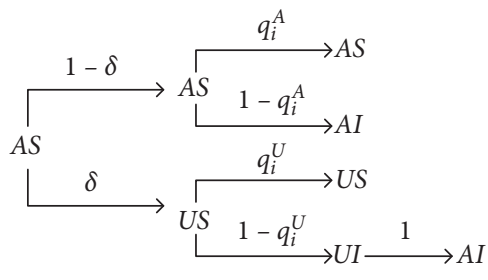

(b)

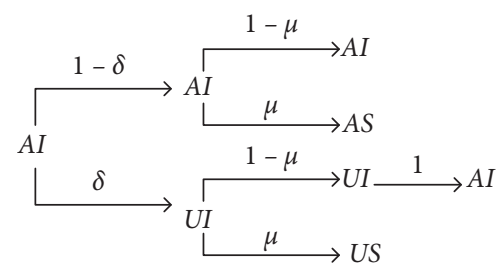

(c)

FIGURE 3: Probability trees for the transition for the possible states at each time step for the model. The four possible states are aware-infected $(A I)$, aware-susceptible $(A S)$, unaware-infected $(U I)$, and unaware-susceptible (US). The transition probabilities are $r_{i}$ from $U$ to $A, q_{i}^{A}$ from $S$ to $I$ if node $i$ is aware, $q_{i}^{U}$ from $S$ to $I$ if node $i$ is unaware, $\delta$ from $A$ to $U$, and $\mu$ from $I$ to $S$.

Figures 4 and 5 show an intersection of $\rho_{A}$ in the middle range of $R_{0}$. For small $R_{0}$, the population with awareness $\rho_{A}$ for high vigilance (larger $x$ in Figures 4 and 5 ) is lower than that for low vigilance, and it reverses when $R_{0}$ becomes large. This implies that high vigilance has a stronger suppressive effect on the spread of the epidemic but does not always result in a lower ratio of $\rho_{A}$ owing to the containment of the epidemic. For small $R_{0}$, high vigilance can suppress the spread of both epidemic and information, whereas, for large $R_{0}$, high vigilance can suppress the epidemic but can slightly facilitate the spread of information. This can be understood from the first equation in equation (4). The stationary state $p_{i}^{U S}(t+1)=p_{i}^{U S}(t)$ yields $p_{i}^{U S}=\left(\left(p_{i}^{A I} \delta \mu+p_{i}^{A S} \delta q_{i}^{U}\right) /\left(1-r_{i} q_{i}^{U}\right)\right)$. In the case of a low infection rate, the infected population is small $\left(\rho_{I} \ll 1\right)$, and $p_{i}^{A I} \ll p_{i}^{A S}$ leads to $p_{i}^{U S} \approx\left(\left(p_{i}^{A S} \delta q_{i}^{U}\right) /\left(1-r_{i} q_{i}^{U}\right)\right)$. The term $r_{i} q_{i}^{U}$ in the denominator is a high-order small quantity compared with the numerator, and the fluctuation of $p_{i}^{U S}$ primarily depends on the fluctuation of $p_{i}^{A S}$ and $q_{i}^{U}$. When vigilance increases, $\rho_{I}$ tends to decrease, leading to higher $p_{i}^{A S}$ and $q_{i}^{U}$ (estimated from equation (3)). Therefore, a higher vigilance level can lead to a higher $p_{i}^{U S}$, resulting in a decrease in $\rho_{A}$ because of the relation $\rho_{A}=1-\sum_{i} p_{i}^{U S}$. In the case of a high infection rate, $p_{i}^{A I} \gg p_{i}^{A S}$ and thus $p_{i}^{U S}=\left(p_{i}^{A I} \delta \mu /\left(1-r_{i} q_{i}^{U}\right)\right)$, where the fluctuation of $p_{i}^{U S}$ primarily depends on the fluctuation of $p_{i}^{A I}$. For a higher vigilance level, $p_{i}^{A I}$ tends to decrease, resulting in a decrease in $p_{i}^{U S}$ and, thus, an increase in $\rho_{A}$. These different behaviors for small and large $R_{0}$ underlie the observed intersection of $\rho_{A}$ for different vigilance levels in the intermediate range of the infection rate.

We have demonstrated that the dependence of $\gamma$ on $\rho_{A}$ can assist in suppressing the spread of the epidemic, assuming that all individuals are rational-higher prevalence of the discussion about the disease leads to a higher level of vigilance. However, in reality, not all individuals are rational. Irrational behaviors, such as irrational beliefs [39], irrational antimicrobial prescribing [39], and panic buying [40], may facilitate the spread of the epidemic by inducing individuals to take improper protective measures. Therefore, we now consider cases in which a part of the population is irrational. As irrational behavior can lead to the opposite effects of those by rational behavior, in our model, these two types of individuals are defined as follows:
Dependence 1: $\gamma=\left(1-\rho_{A}\right)^{x}$ for rational individuals, and $\gamma=\left(1+\rho_{A}\right)^{x}$ for irrational individuals

Dependence 2: $\gamma=x^{-\rho_{A}}$ for rational individuals, and $\gamma=x^{\rho_{A}}$ for irrational individuals

Accordingly, disease awareness can reduce the infection rate for rational individuals but increase the infection rate for irrational individuals.

Figure 6 shows the effect of irrational individuals on the spread of the epidemic for the first form of dependence. As the irrational population increases, the final infected population increases, whereas the critical infection rate decreases. This indicates that irrational individuals can facilitate the spread of the epidemic by introducing a negative correlation between vigilance and awareness prevalence. In extreme cases in which irrational individuals become the majority of the system, the spread of information can no longer suppress the epidemic. As shown in Figures 6(d) and 6(f), if most individuals are irrational and take improper measures, a larger population with awareness leads to more serious outbreaks of the epidemic. A similar conclusion can also be obtained for the second form of dependence, as shown in Figure 7. Heterogeneity is an important characteristic of real contagion networks, and different spatial distributions may impact the effect of irrational individuals. Thus, we also performed numerical simulations on BA networks. Figure 8 shows the result of the first form of dependence for three different infection rates: $R_{0}=1.25,2.5$, and 3.75. The irrational individuals are distributed on nodes with the highest degree, nodes with the lowest degree, and randomly chosen nodes. It can be concluded that, in the second case, the epidemic is not significantly worsened by a small fraction of irrational individuals. When the irrational individuals are randomly distributed or placed on nodes with a high degree, the final infected population increases. This effect of irrational individuals is more pronounced for smaller values of $R_{0}$. Figure 8(d) shows the dependence of the critical infection rate on the ratio of irrational individuals for various scenarios. Again, we observe that an increased ratio of irrational individuals can facilitate the spread of the epidemic. A similar effect is observed for the second form of independence in Figure 9. 


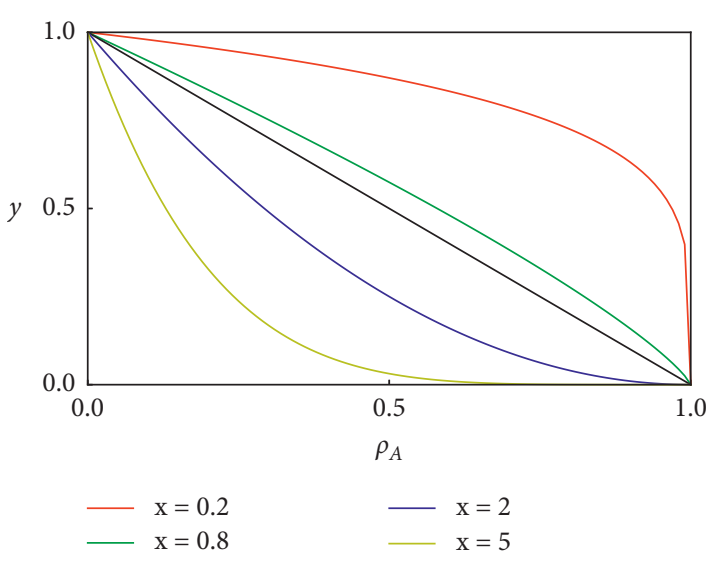

(a)

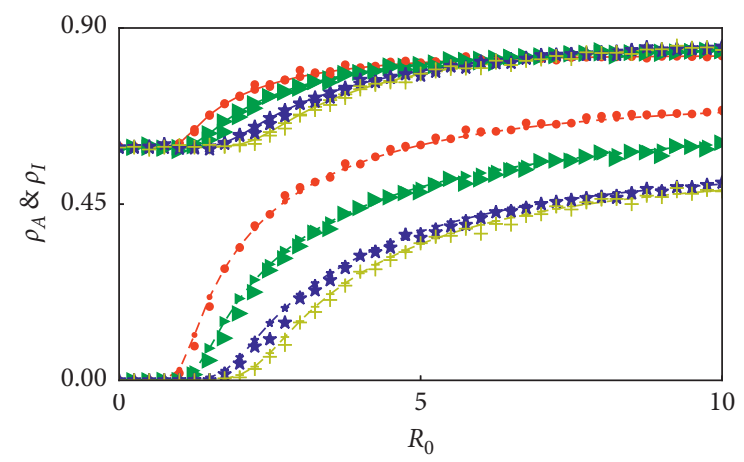

$\begin{aligned} \rightarrow \mathrm{x}=0.2 & \star \mathrm{x}=2 \\ \rightarrow \mathrm{x}=0.8 & +\mathrm{x}=5\end{aligned}$

(c)

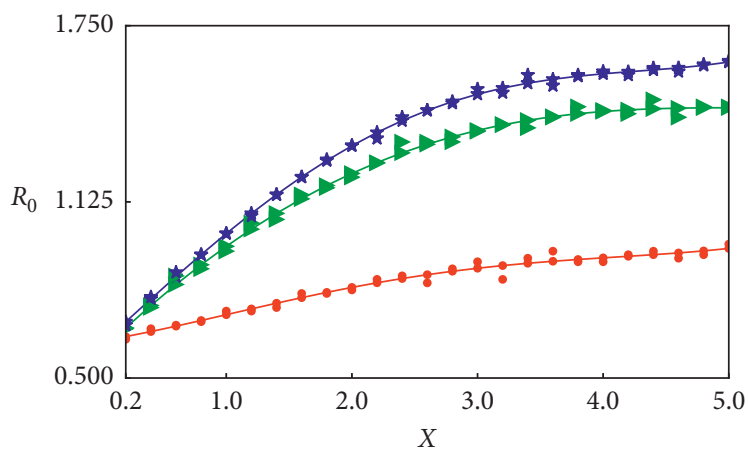

$$
\begin{aligned}
& \multimap \lambda=0.1 \\
& \rightarrow \lambda=0.3 \\
& \star \lambda=0.8
\end{aligned}
$$

(e)

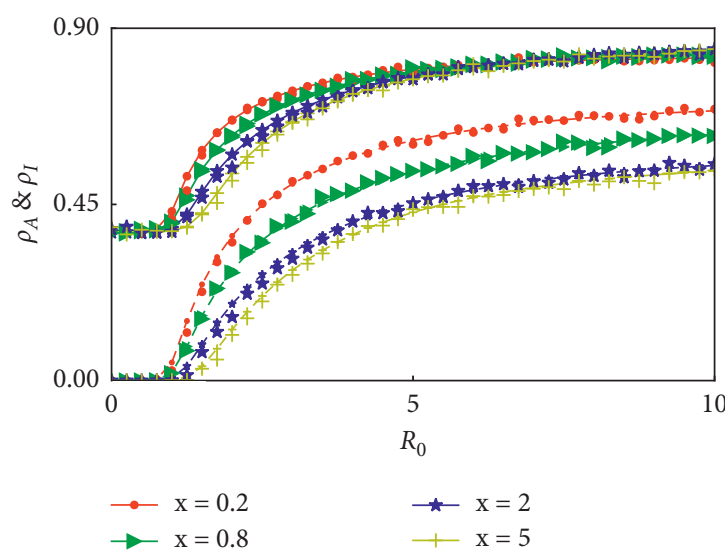

(b)

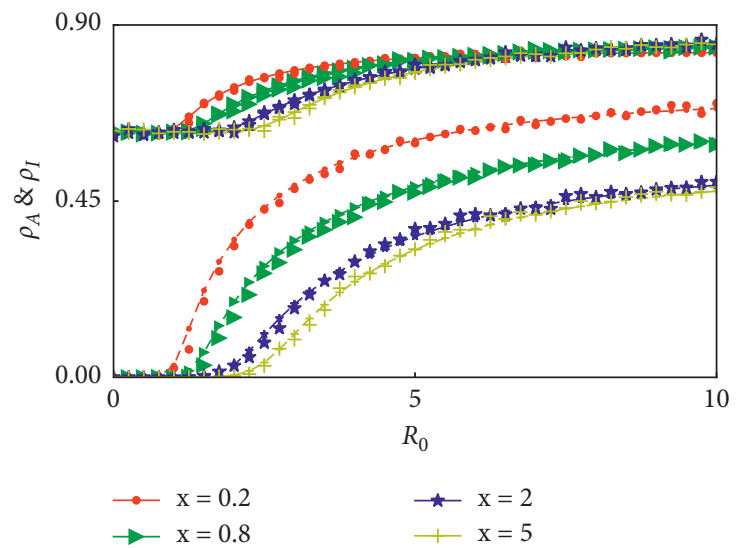

(d)

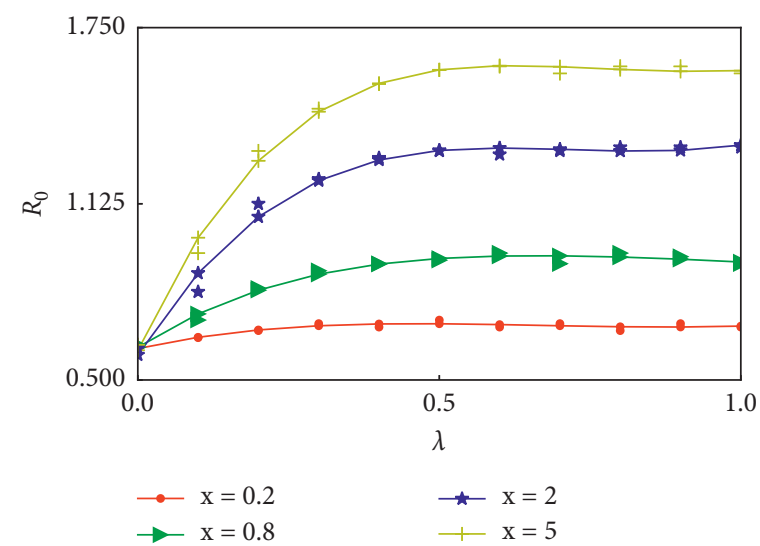

(f)

Figure 4: (a) Plots of the function $\gamma=\left(1-\rho_{A}\right)^{x}$ for different $x$ values. The dependence of prevalence $\rho_{A}$ and $\rho_{I}$ on the infection rate $R_{0}$ is shown in (b) for $\lambda=0.1$, (c) for $\lambda=0.3$, and (d) for $\lambda=0.8$. Solid lines represent the ratio of awareness nodes $\rho_{A}$ and dashed lines represent the ratio of infected nodes. Symbols represent the simulation results and different colors correspond to different $x$ values. (e) Dependence of the critical infection rate $R_{0 c}$ for the epidemic outbreak on parameter $x$. (f) Dependence of $R_{0 c}$ on $\lambda$, with $\delta=0.6$ and $\mu=0.4$. 


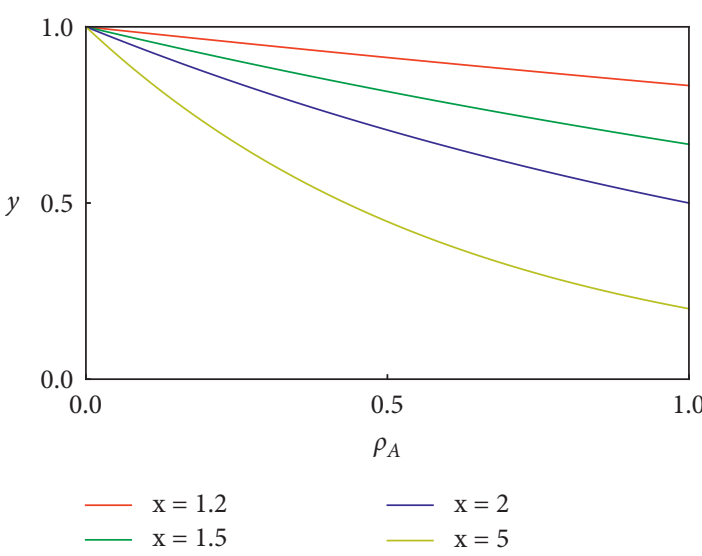

(a)

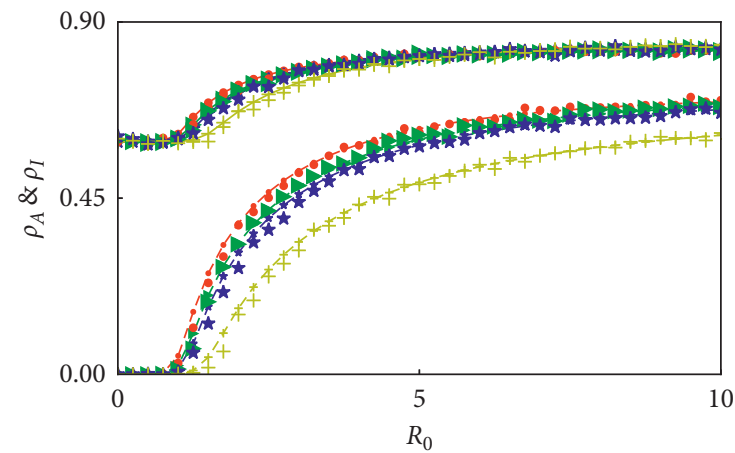

$\rightarrow \mathrm{x}=1.2$

$\rightarrow \mathrm{x}=1.5$

* $\mathrm{x}=2$

(c)

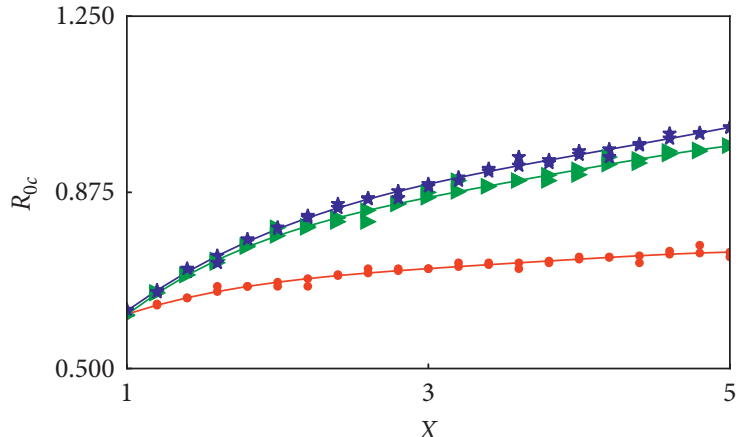

$\rightarrow \lambda=0.1$
$\rightarrow \lambda=0.3$
$\star \lambda=0.8$
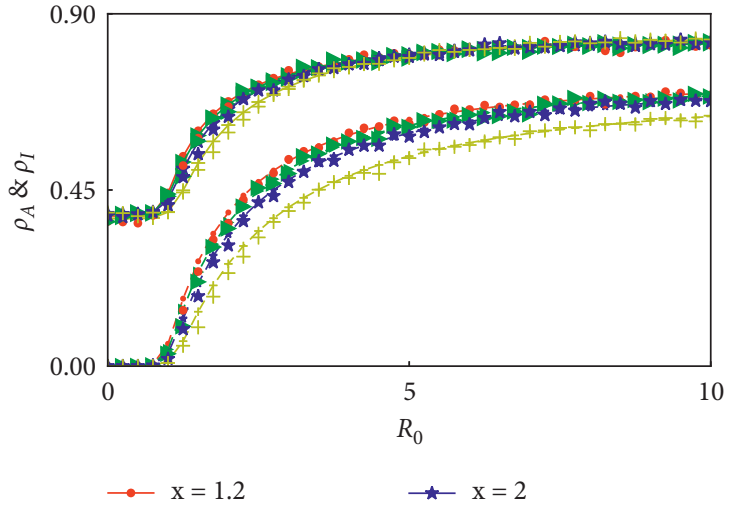

$\rightarrow \mathrm{x}=1.5$

(b)
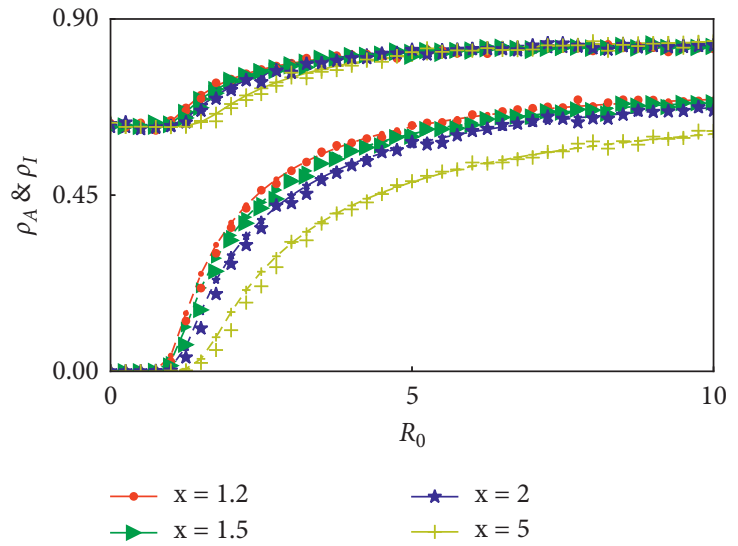

(d)

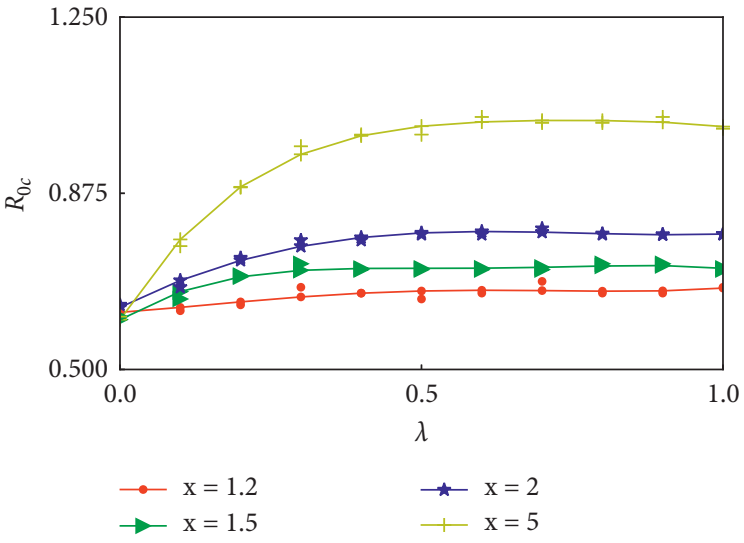

(f)

Figure 5: (a) Plots of the function $\gamma=x^{-\rho_{A}}$ for different $x$ values. The dependence of prevalence $\rho_{A}$ and prevalence $\rho_{I}$ on the infection rate $R_{0}$ is shown in (b) for $\lambda=0.1$, (c) for $\lambda=0.3$, and (d) for $\lambda=0.8$. Solid lines represent the ratio of awareness nodes $\rho_{A}$ and dashed lines represent the ratio of infected nodes. Symbols represent the simulation results and different colors correspond to different $x$ values. (e) Dependence of the critical infection rate $R_{0 c}$ for the epidemic outbreak on parameter $x$. (f) Dependence of $R_{0 c}$ on $\lambda$, with $\delta=0.6$ and $\mu=0.4$. 


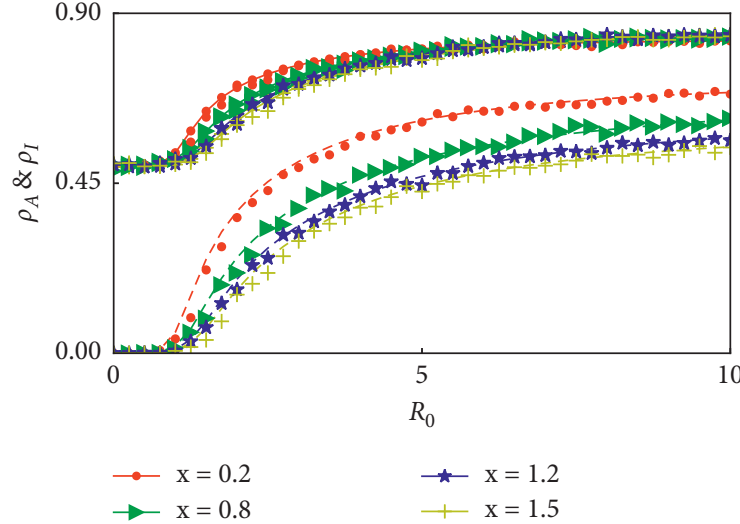

(a)

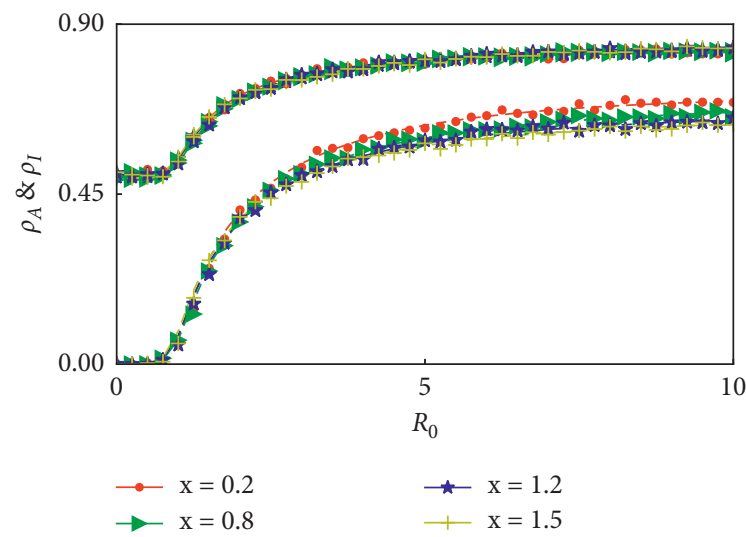

(c)

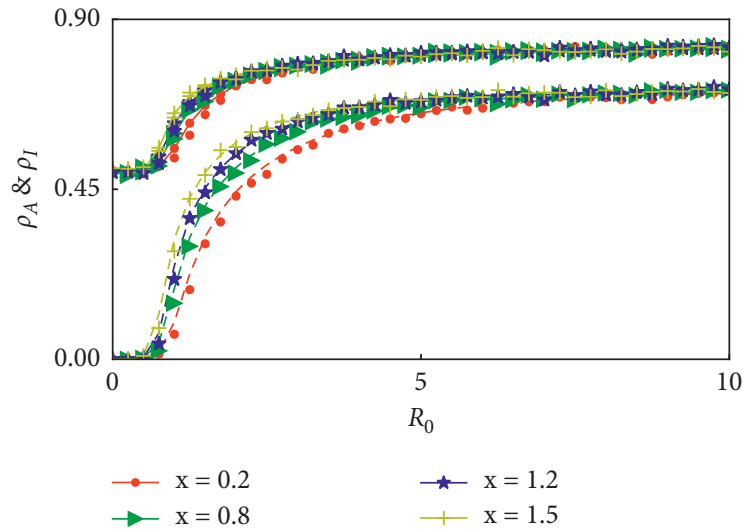

(e)

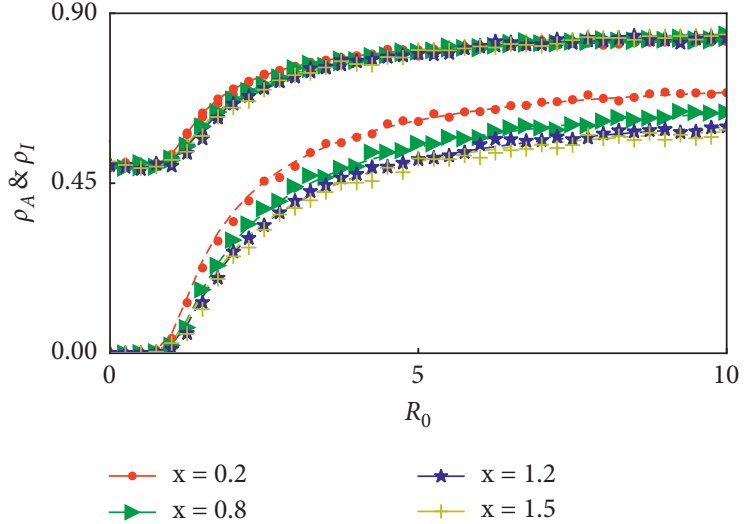

(b)

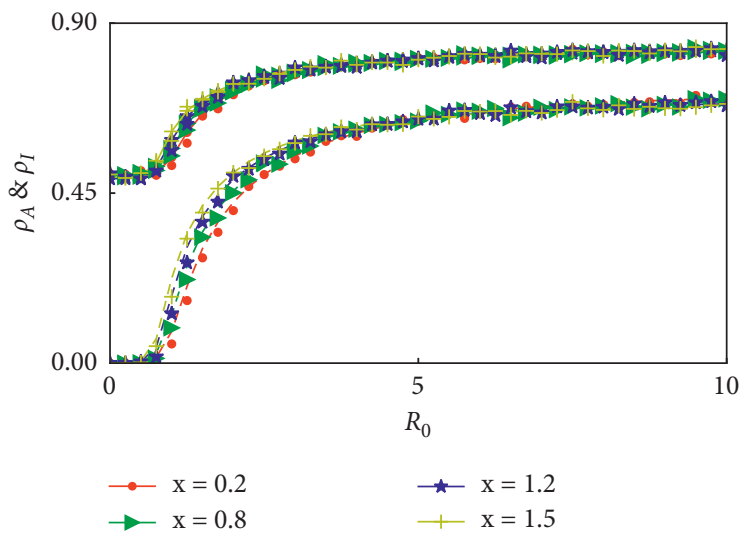

(d)

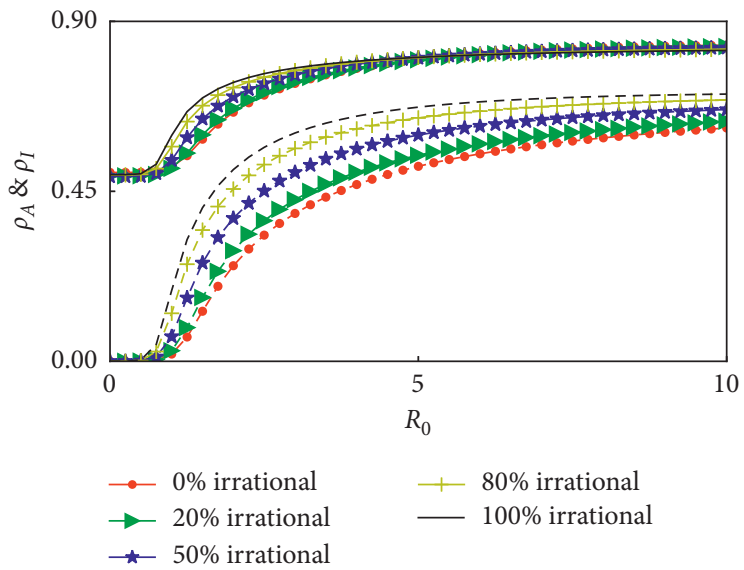

(f)

Figure 6: For the first form of dependence, where $\gamma=\left(1-\rho_{A}\right)^{x}$ for rational individuals and $\gamma=\left(1+\rho_{A}\right)^{x}$ for irrational individuals, the dependence of prevalence $\rho_{A}$ and prevalence $\rho_{I}$ on the infection rate $R_{0}$ is calculated for (a) $0 \%$, (b) $20 \%$, (c) $50 \%$, (d) $80 \%$, and (e) $100 \%$ irrational individuals. Solid lines represent the ratio of awareness nodes $\rho_{A}$ and dashed lines represent the ratio of infected nodes. Symbols represent the simulation results and different colors correspond to different $x$ values. (f) Comparison between cases of different ratios of irrational individuals, with $x=0.8$. All calculations are based on $\delta=0.6$ and $\mu=0.4$. 


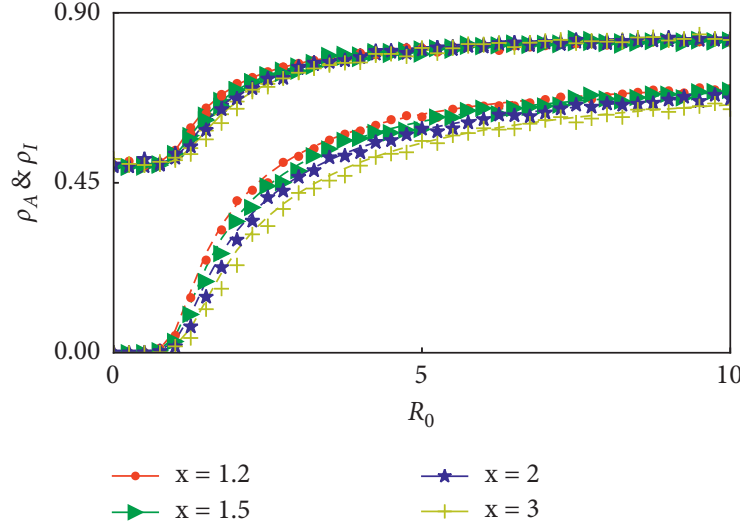

(a)

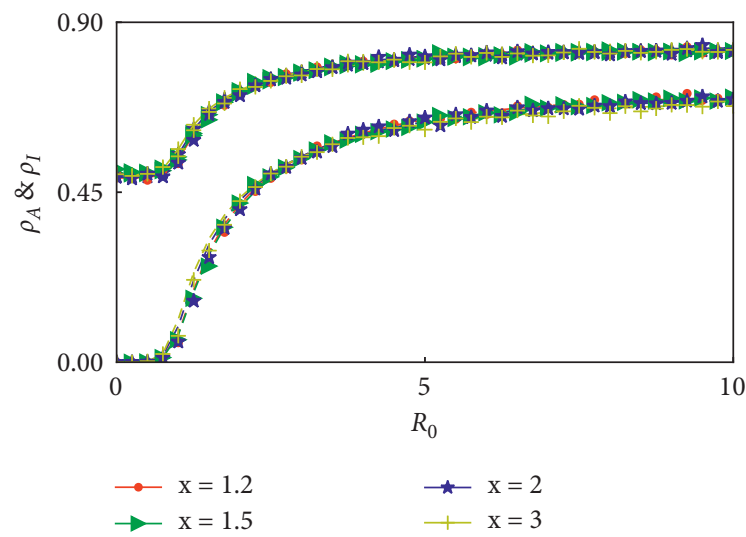

(c)

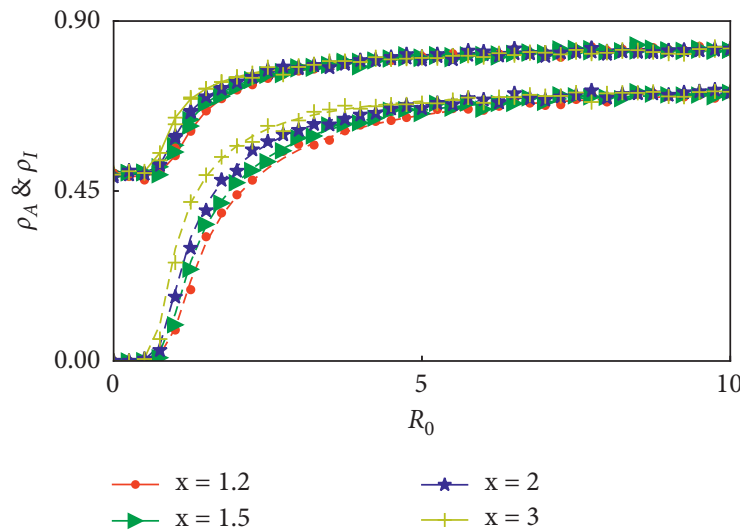

(e)

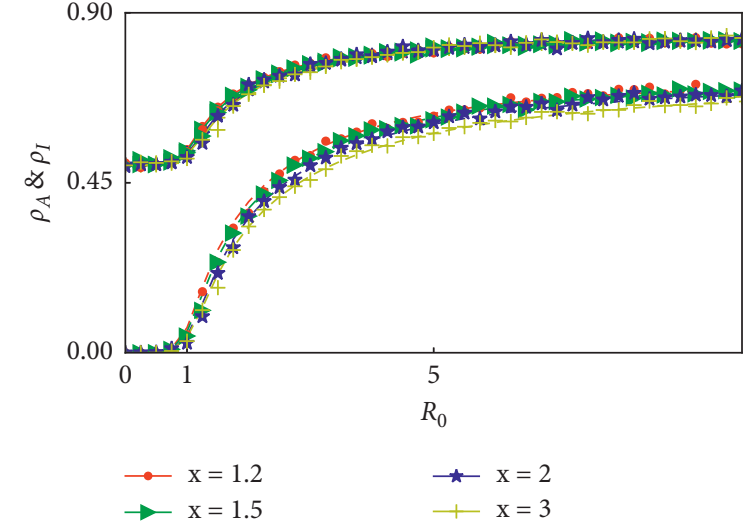

(b)

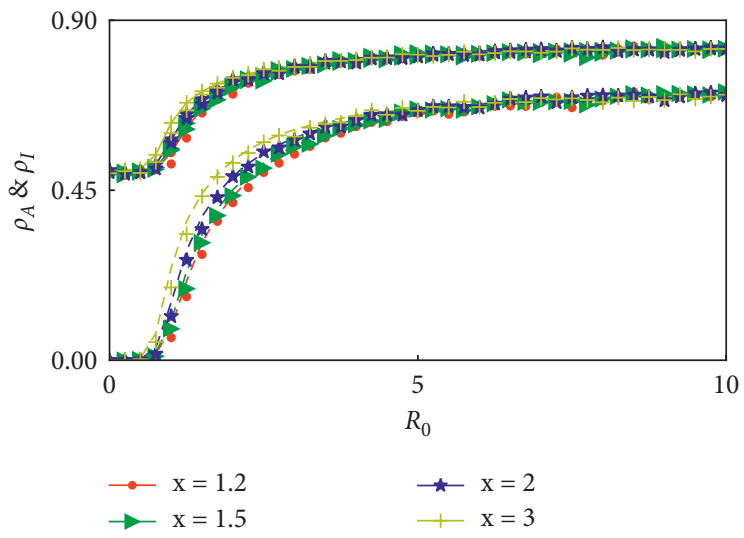

(d)

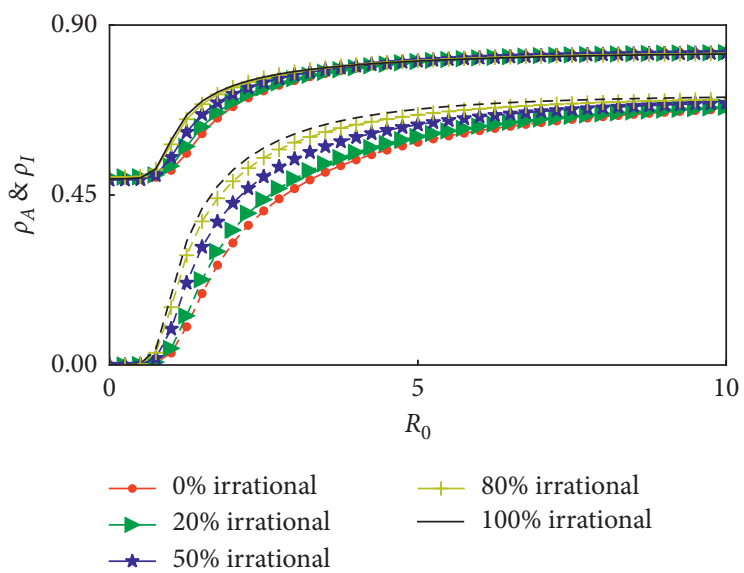

(f)

FIgURE 7: For the second form of dependence, where $\gamma=x^{-\rho_{A}}$ for rational individuals and $\gamma=x^{\rho_{A}}$ for irrational individuals, the dependence of prevalence $\rho_{A}$ and prevalence $\rho_{I}$ on the infection rate $R_{0}$ is calculated for (a) $0 \%$, (b) 20\%, (c) 50\%, (d) $80 \%$, and (e) $100 \%$ irrational individuals, respectively. Solid lines represent the ratio of awareness nodes $\rho_{A}$ and dashed lines represent the ratio of infected nodes. Symbols represent the simulation results and different colors correspond to different $x$ values. (f) Comparison between cases of different ratios of irrational individuals, with $x=2$. All calculations are based on $\delta=0.6$ and $\mu=0.4$. 

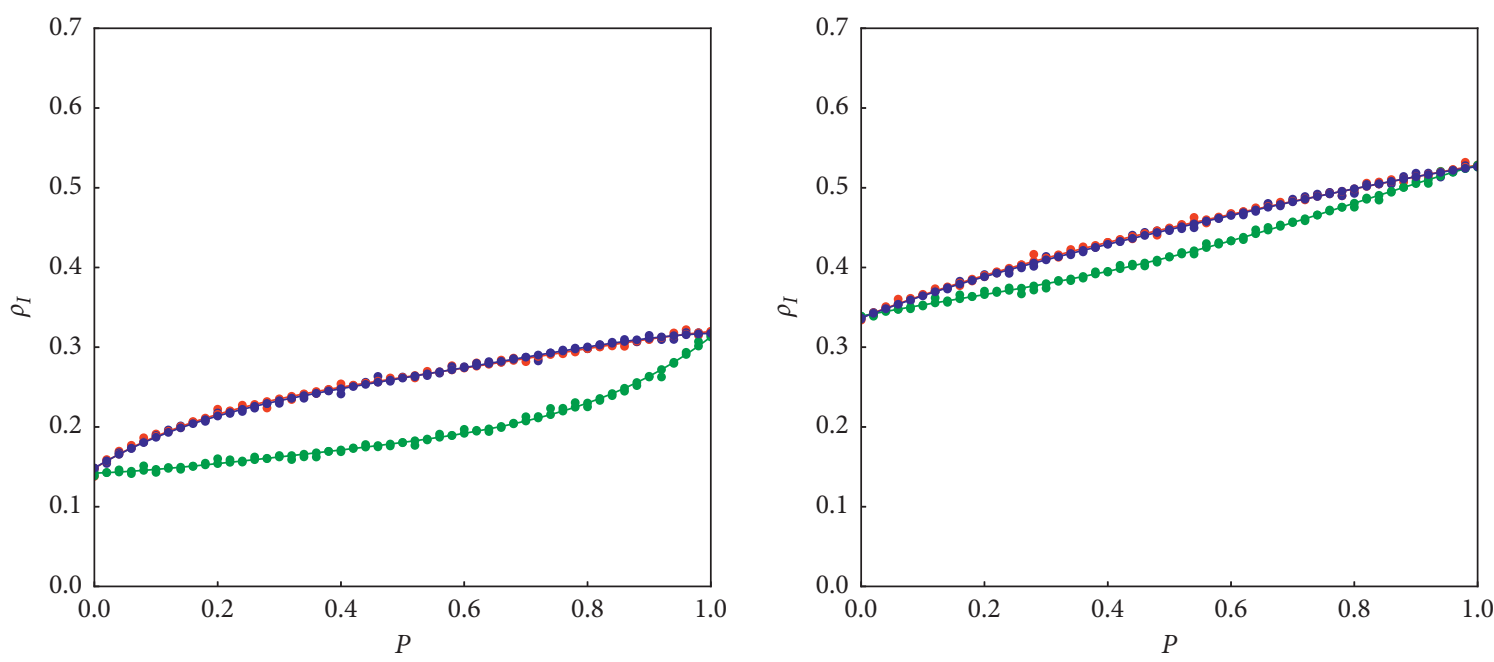

$$
\begin{aligned}
& \longrightarrow \text { High degree } \\
& \because \text { Low degree } \\
& \rightarrow \text { Random }
\end{aligned}
$$

(a)

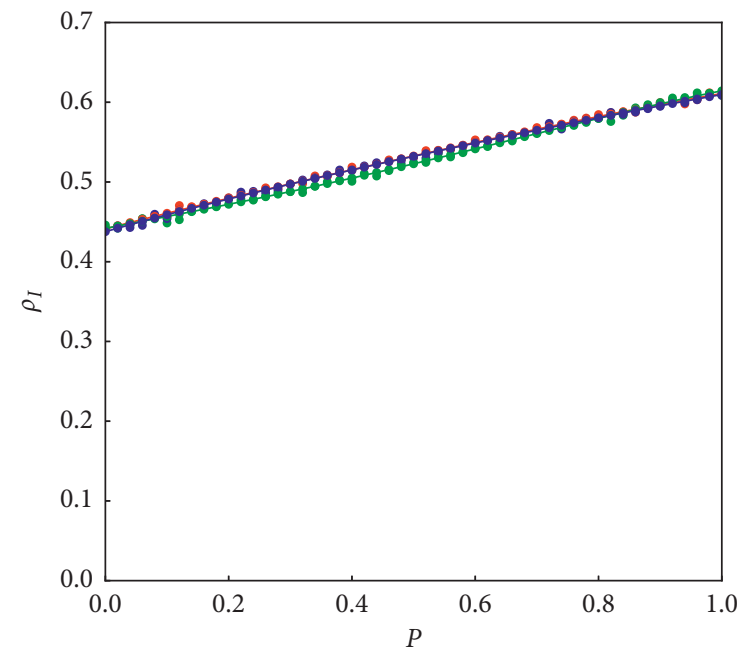

$\rightarrow$ High degree

$\rightarrow$ Low degree

$\rightarrow$ Random

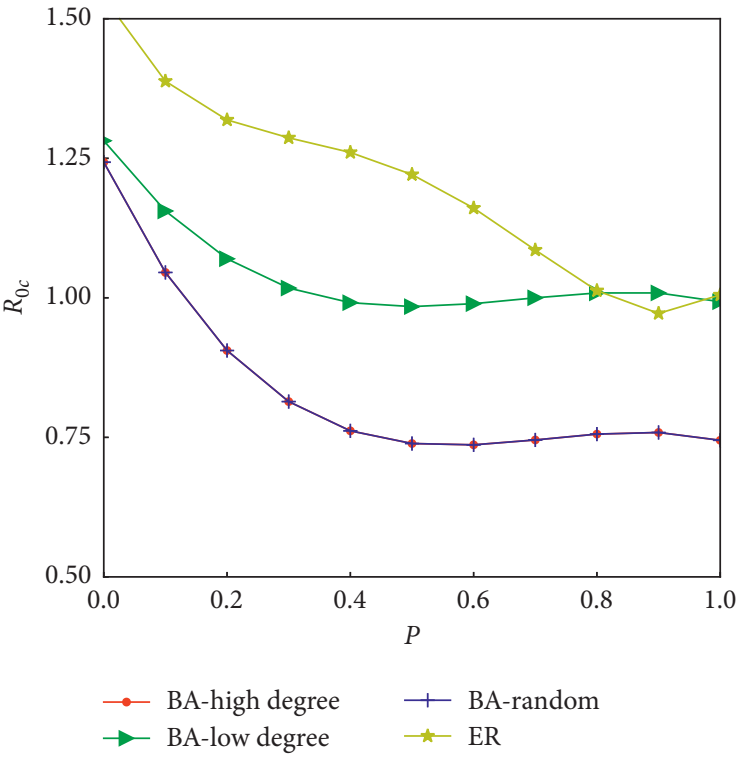

(d)

Figure 8: For the first form of dependence, where $\gamma=\left(1-\rho_{A}\right)^{x}$ for rational individuals and $\gamma=\left(1+\rho_{A}\right)^{x}$ for irrational individuals, the dependence of epidemic prevalence $\rho_{I}$ on the ratio of irrational individuals on the BA scale-free network for (a) $R_{0}=1.25$, (b) $R_{0}=2.5$, and (c) $R_{0}=3.75$. Irrational individuals are distributed according to three different strategies: on nodes with the highest degree (red line), on nodes with the lowest degree (green line), and randomly distributed (blue line). (d) The critical infection rate $R_{0 c}$ for epidemic outbreak in different situations. The parameters are the same as those in Figures 4 and 6. 

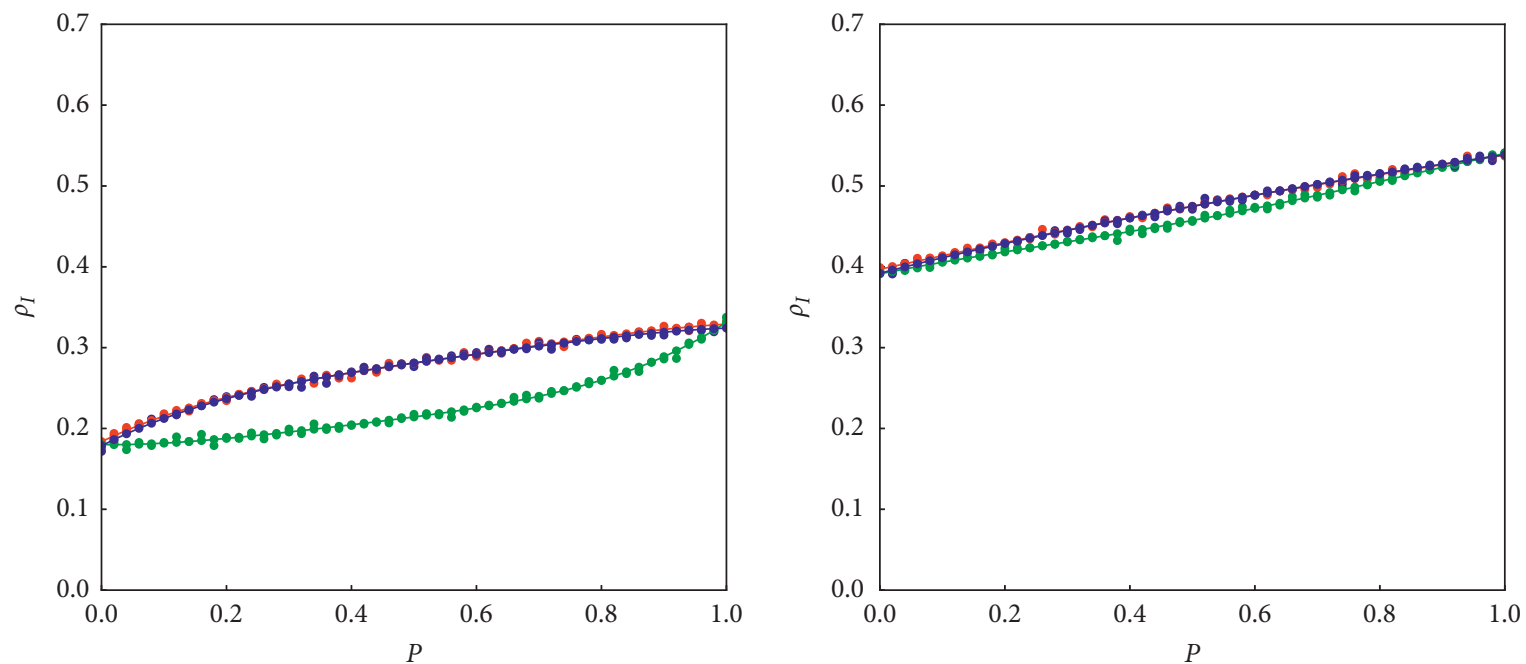

$\rightarrow$ High degree

$\rightarrow$ High degree

- Low degree

$\rightarrow$ Low degree

$\rightarrow$ Random

$\rightarrow$ Random

(a)
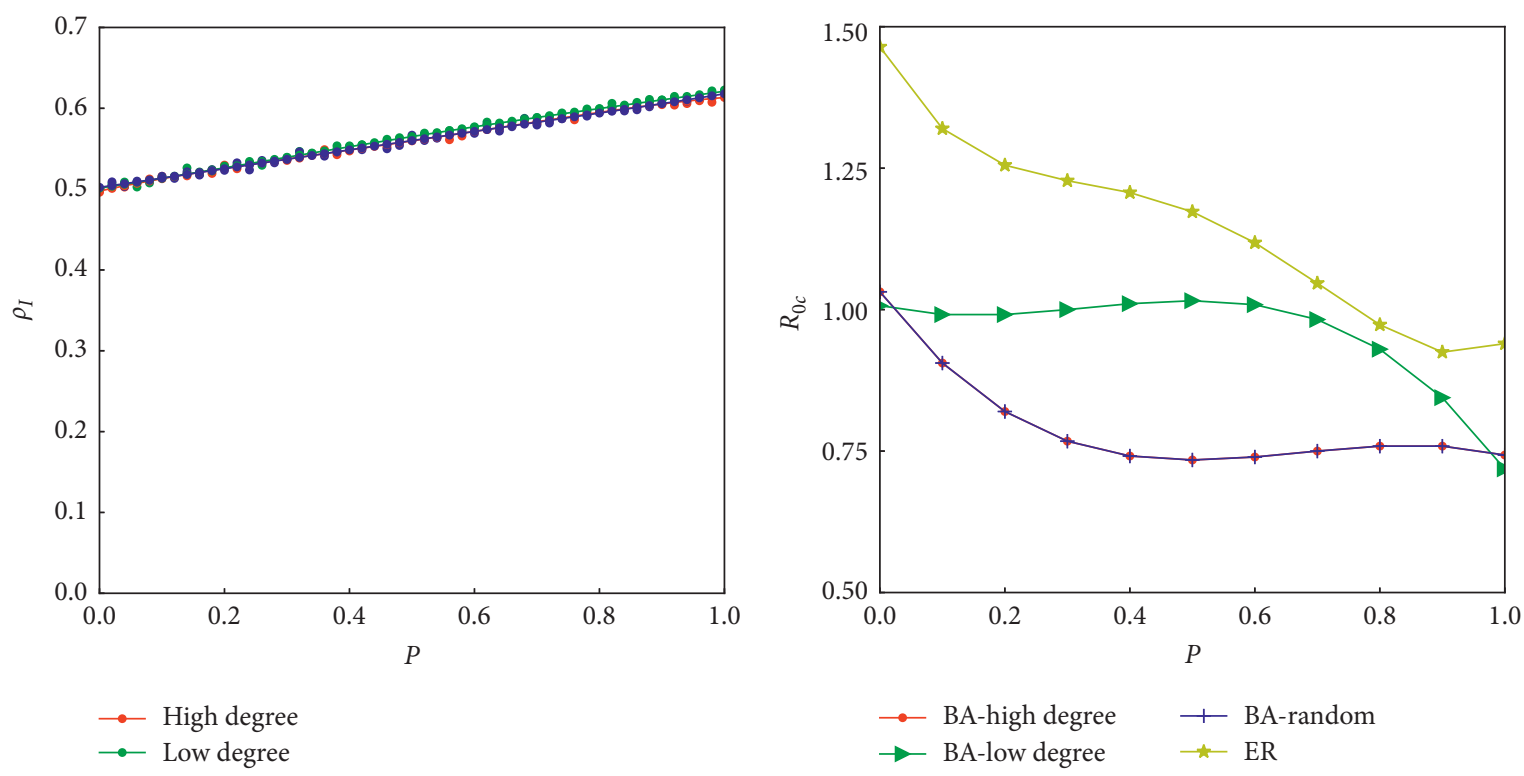

(c)

(d)

FIGURE 9: For the first form of dependence, where $\gamma=x^{-\rho_{A}}$ for rational individuals and $\gamma=x^{\rho_{A}}$ for irrational individuals, the dependence of epidemic prevalence $\rho_{I}$ on the ratio of irrational individuals in the BA scale-free network for (a) $R_{0}=1.25$, (b) $R_{0}=2.5$, and (c) $R_{0}=3.75$. Irrational individuals are distributed according to three different strategies: on nodes with the highest degree (red line), on nodes with the lowest degree (green line), and randomly distributed (blue line). (d) The critical infection rate $R_{0 c}$ for epidemic outbreak in different situations. The parameters are the same as those in Figures 4 and 6. 
These results demonstrate the importance of maintaining rationality in containing epidemics, as the efficiency of the strategy of inducing individuals to become aware of the disease and take protective measures depends on the ratio of individuals who can take proper measures. If most individuals cannot take proper protective measures, the spread of awareness cannot assist in containing the epidemic. Thus, irrational population is also a factor that may affect the spread of the pandemic.

\section{Conclusion}

In modern society, online communication and social media have become important sources of information. Accordingly, relevant information can be spread faster during a pandemic. Individuals can become vigilant about the spread of the disease and take protective measures. Therefore, the process of information propagation can suppress epidemics to a certain extent. In a more realistic scenario, the vigilance of individuals who are aware of the disease is not constant. The level of vigilance and protective measures may change with awareness prevalence. Therefore, investigating the effect of the dependence of individual vigilance on awareness prevalence may lead to a deeper understanding of the coevolution dynamics between epidemic spreading and information spreading.

In this study, we investigated the effect of various forms of dependence between individual vigilance and the spread of awareness of a disease. For rational individuals, vigilance and the level of protective measures increase with the prevalence of discussion. We demonstrated that this positive dependence can enhance the effect of information spreading in suppressing epidemics, as shown in Figures 4 and 5. However, individuals may not be rational and may not take proper protective measures. These irrational behaviors may weaken the effect of information spreading in the containment of the epidemic. In extreme cases where most individuals are irrational, information propagation may even facilitate the spread of the disease, as shown in Figures 6-9. Thus, our results demonstrate the importance of rational behaviors in containing epidemics. Information propagation can efficiently suppress epidemics only when most individuals remain rational and can take proper protective measures.

\section{Data Availability}

The data that support the findings of this study are available from the corresponding author upon reasonable request.

\section{Conflicts of Interest}

The authors declare that there are no conflicts of interest regarding the publication of this paper.

\section{Acknowledgments}

This study was supported by the National Social Science Foundation of China, "Risk Communication and Effectiveness Evaluation in Public Crises" (20AXW008).

\section{References}

[1] C. Castellano, P. Van Mieghem, and A. Vespignani, "Epidemic processes in complex networks," Reviews of Modern Physics, vol. 87, no. 3, pp. 925-979, 2015.

[2] U. Harush and B. Barzel, "Dynamic patterns of information flow in complex networks," Nature Communications, vol. 8, Article ID 2181, 2017.

[3] C. Hens, U. Harush, S. Haber, R. Cohen, and B. Barzel, "Spatiotemporal signal propagation in complex networks," Nature Physics, vol. 15, no. 4, pp. 403-412, 2019.

[4] Q. Liu, W. Wang, M. Tang, T. Zhou, and Y. Lai, "Explosive spreading on complex networks: the role of synergy," Physical Review E, vol. 95, Article ID 042320, 2017.

[5] J. Wu, M. Zheng, K. Xu, and C. Gu, "Effects of two channels on explosive information spreading," Nonlinear Dynamics, vol. 99, no. 3, pp. 2387-2397, 2020.

[6] L. Hébert-Dufresne, S. V. Scarpino, and J.-G. Young, "Macroscopic patterns of interacting contagions are indistinguishable from social reinforcement," Nature Physics, vol. 16 , no. 4 , pp. 426-431, 2020.

[7] T. Gross, C. J. D. Lima, and B. Blasius, "Epidemic dynamics on an adaptive network," Physical Review Letters, vol. 96, Article ID 208701, 2006.

[8] L. Böttcher, O. Woolley-Meza, E. Goles, D. Helbing, and H. J. Herrmann, "Connectivity disruption sparks explosive epidemic spreading," Physical Review E, vol. 93, Article ID 042315, 2016.

[9] X. Zhang, Z. Ruan, M. Zheng, B. Barzel, and S. Boccaletti, "Epidemic spreading under infection-reduced-recovery," Chaos, Solitons \& Fractals, vol. 140, Article ID 110130, 2020.

[10] L. Pan, W. Wang, L. Tian, and Y. C. Lai, "Optimal networks for dynamical spreading," Physical Review E, vol. 103, Article ID 012302, 2021.

[11] L. Pan, W. Wang, S. Cai, and T. Zhou, "Optimal interlayer structure for promoting spreading of the susceptible-infectedsusceptible model in two-layer networks," Physical Review E, vol. 100, Article ID 022316, 2019.

[12] L. Pan, D. Yang, W. Wang, S. Cai, T. Zhou, and Y. C. Lai, "Phase diagrams of interacting spreading dynamics in complex networks," Physical Review Research, vol. 2, Article ID 023233, 2020.

[13] V. Colizza, A. Barrat, M. Barthélemy, and A. Vespignani, "The role of the airline transportation network in the prediction and predictability of global epidemics," Proceedings of the National Academy of Sciences of the USA, vol. 103, Article ID 2015, 2005.

[14] V. Colizza, A. Barrat, M. Barthélemy, and A. Vespignani, "Epidemic predictions and predictability in complex environments," Biophysical Reviews and Letters, vol. 03, no. 01n02, pp. 217-226, 2008.

[15] C. Dye and N. Gay, "EPIDEMIOLOGY: modeling the SARS epidemic," Science, vol. 300, no. 5627, pp. 1884-1885, 2003.

[16] K. Khan, J. Arino, W. Hu et al., "Spread of a novel influenza A (H1N1) virus via global airline transportation," New England Journal of Medicine, vol. 361, no. 2, pp. 212-214, 2009.

[17] P. Mukherjee, P. L. Lim, A. Chow et al., "Epidemiology of travel-associated pandemic $\left(\mathrm{H}_{1} \mathrm{~N}_{1}\right) 2009$ infection in 116 patients, Singapore," Emerging Infectious Diseases, vol. 16, no. 1, pp. 21-26, 2010.

[18] P. Bajardi, C. Poletto, J. J. Ramasco, M. Tizzoni, V. Colizza, and A. Vespignani, "Human mobility networks, travel restrictions, and the global spread of $2009 \mathrm{H}_{1} \mathrm{~N}_{1}$ pandemic," PLoS One, vol. 6, Article ID e16591, 2011. 
[19] Z. Ruan, M. Tang, and Z. Liu, "How the contagion at links influences epidemic spreading," The European Physical Journal B, vol. 86, p. 149, 2013.

[20] X. Pang, L. Ren, S. Wu et al., "COVID-19 field response group, COVID-19 laboratory testing group, "cold-chain food contamination as the possible origin of COVID-19 resurgence in Beijing," National Science Review, vol. 7, no. 12, pp. 1861-1864, 2020.

[21] P. Manfredi and A. D'Onofrio, Modeling the Interplay between Human Behavior and the Spread of Infectious Diseases, Springer-Verlag, Berlin, Germany, 2013.

[22] S. Funk, M. Salathé, and V. A. A. Jansen, "Modelling the influence of human behaviour on the spread of infectious diseases: a review," Journal of the Royal Society Interface, vol. 7, Article ID 1257, 2010.

[23] S. Funk, E. Gilad, and V. A. A. Jansen, "Endemic disease, awareness, and local behavioural response," Journal of Theoretical Biology, vol. 264, no. 2, pp. 501-509, 2010.

[24] Z. Ruan, M. Tang, and Z. Liu, "Epidemic spreading with information-driven vaccination," Physical Review E, vol. 86, Article ID 036117, 2012.

[25] S. Funk, E. Gilad, C. Watkins, and V. A. A. Jansen, "The spread of awareness and its impact on epidemic outbreaks," Proceedings of the National Academy of Sciences, vol. 106, no. 16, pp. 6872-6877, 2009.

[26] S. Funk and V. A. A. Jansen, "Interacting epidemics on overlay networks," Physical Review E, vol. 81, Article ID 036118, 2010.

[27] X.-X. Zhan, C. Liu, G.-Q. Sun, and Z.-K. Zhang, "Epidemic dynamics on information-driven adaptive networks," Chaos, Solitons \& Fractals, vol. 108, pp. 196-204, 2018.

[28] Q. Wu, X. Fu, M. Small, and X. Xu, "The impact of awareness on epidemic spreading in networks," Chaos, vol. 22, Article ID 013101, 2012.

[29] X.-X. Zhan, C. Liu, G. Zhou et al., "Coupling dynamics of epidemic spreading and information diffusion on complex networks," Applied Mathematics and Computation, vol. 332, pp. 437-448, 2018.

[30] C. Granell, S. Gómez, and A. Arenas, "Dynamical interplay between awareness and epidemic spreading in multiplex networks," Physical Review Letters, vol. 111, Article ID 128701, 2013.

[31] C. Granell, S. Gómez, and A. Arenas, "Competing spreading processes on multiplex networks: awareness and epidemics," Physical Review E, vol. 90, Article ID 012808, 2014.

[32] W. Wang, M. Tang, H. Yang, Y. Do, Y. Lai, and G. Lee, "Asymmetrically interacting spreading dynamics on complex layered networks," Scientific Reports, vol. 4, p. 5097, 2014.

[33] W. Wang, Q. Liu, S. Cai, M. Tang, L. A. Braunstein, and H. E. Stanley, "Suppressing disease spreading by using information diffusion on multiplex networks," Scientific Reports, vol. 6, Article ID 29259, 2016.

[34] C. Xu, X. Zhang, and Y. Wang, "Mapping of health literacy and social panic via web search data during the COVID-19 public health emergency: infodemiological study," Journal of Medical Internet Research, vol. 22, Article ID e18831, 2020.

[35] A. Depoux, S. Martin, E. Karafillakis, R. Preet, A. WilderSmith, and H. Larson, "The pandemic of social media panic travels faster than the COVID-19 outbreak," Journal of Travel Medicine, vol. 27, no. 3, Article ID taaa031, 2020.

[36] A. R. Ahmad and H. R. Murad, "The impact of social media on panic during the COVID-19 pandemic in Iraqi Kurdistan: online questionnaire study," Journal of Medical Internet Research, vol. 22, Article ID e19556, 2020.
[37] E. Dong, H. Du, and L. Gardner, "An interactive web-based dashboard to track COVID-19 in real time Modeling and forecasting of epidemic spreading: the case of COVID-19 and beyond," Lancet Infect Disease, vol. 3099, pp. 19-20, 2020.

[38] P. Teovanović, P. Lukić, Z. Zupan, A. Lazić, M. Ninković, and I. Žeželj, "Irrational beliefs differentially predict adherence to guidelines and pseudoscientific practices during the COVID19 pandemic," Applied Cognitive Psychology, vol. 35, pp. 486-496, 2020.

[39] M. Parveen, M. Yeasmin, and M. A. Molla, “Antimicrobial resistance, evidences on irrational anti-microbial prescribing and consumption during COVID-19 pandemic and possible mitigation strategies: a Bangladesh perspective," Bangladesh Journal of Infectious Diseases, vol. 7, pp. S3-SX7, 2020.

[40] S. M. Y. Arafat, S. K. Kar, V. Menon et al., "Panic buying: an insight from the content analysis of media reports during COVID-19 pandemic," Neurology, Psychiatry and Brain Research, vol. 37, pp. 100-103, 2020.

[41] D. Chakrabarti, Y. Wang, C. Wang, J. Leskovec, and C. Faloutsos, "Epidemic thresholds in real networks," ACM Transactions on Information and System Security, vol. 10, no. 4, pp. 1-26, 2008.

[42] S. Gómez, A. Arenas, J. Borge-Holthoefer, S. Meloni, and Y. Moreno, "Discrete-time Markov chain approach to contact-based disease spreading in complex networks," EPL (Europhysics Letters), vol. 89, no. 3, Article ID 38009, 2010.

[43] S. Gómez, J. Gómez-Gardenës, Y. Moreno, and A. Arenas, "Nonperturbative heterogeneous mean-field approach to epidemic spreading in complex networks," Physical Review E, vol. 84, Article ID 036105, 2011. 